\title{
Laparoscopia nas hérnias diafragmáticas: estudo experimental em cães
}

\author{
Laparoscopic techniques in diaphragmatic hernias: experimental study in dogs
}

\author{
Carlos Afonso de Castro Beck ${ }^{1}$ Ney Luis Pippi ${ }^{2}$ Maurício Veloso Brun ${ }^{3}$ Emerson Antônio Contesini $^{1}$ \\ Anderson Favaro da Cunha ${ }^{5}$ Rafael Stedile $^{5}$ Adamas Tassinari Bonfada $^{5}$ \\ Antonio de Pádua Ferreira da Silva Filho ${ }^{6}$ Antonio Roberto Pinheiro Vieira Júnior ${ }^{7}$ \\ Thiago Félix da Silva ${ }^{7}$ Angela Ilha Bordin ${ }^{5}$
}

\section{RESUMO}

A hérnia diafragmática traumática é a forma mais comum entre as hérnias diafragmáticas em cães e gatos e, por tratar-se de uma alteração anatômica, a terapêutica indicada é a sua correção cirúrgica. O presente estudo se propôs a avaliar a cirurgia videolaparoscópica no diagnóstico e tratamento dessa afecção em cães. Na primeira etapa do experimento, os animais foram submetidos à produção de um modelo de hérnia diafragmática. Após o período de uma semana, os cães foram submetidos à segunda etapa experimental por meio do acesso laparoscópico, tendo como finalidade o diagnóstico e a terapêutica cirúrgica. O acesso laparoscópico mostrou-se eficaz tanto na identificação das rupturas e dos deslocamentos como na correção cirúrgica de reposição visceral e rafia diafragmática nos oito cães do experimento.

Palavras-chave: videolaparoscopia, cirurgia, tórax.

\section{ABSTRACT}

The most common type of diaphragmatic hernia in dogs and cats is the traumatic one. As an anatomic alteration, surgery is the elected treatment. The purpose of this study was to evaluate videolaparoscopic surgery in the diagnostic and treatment of diaphragmatic hernia in dogs. In the first phase of the study diaphragmatic hernias were performed in dogs. One week later the dogs were summited to a laparoscopic surgery to make diagnostic and surgical correction. Laparoscopic access showed to be efficient for identification of the defect and visceral displacement and for the surgical treatment.

Key words: videolaparoscopy, surgery, thorax.

\section{INTRODUÇÃO}

A hérnia diafragmática traumática é a forma mais comum entre as hérnias diafragmáticas em cães e gatos (LEVINE, 1987; JOHNSON, 1993; RAISER, 1993), sendo o acidente automobilístico a causa mais freqüente dessas alterações (WILSON et al., 1971; BOUDRIEAU \& MUIR, 1987; LEVINE, 1987), além de quedas, chutes e brigas (BOUDRIEAU \& MUIR, 1987; LEVINE, 1987; HAGE \& IWASAKI, 2001).

Pelo fato da alteração presente nas rupturas diafragmáticas ser de natureza anatômica, o tratamento cirúrgico é o único indicado (RAISER, 1993; HAGE \& IWASAKI, 2001). As abordagens cirúrgicas mais utilizadas na medicina veterinária têm sido: a laparotomia pela linha média e a toracotomia intercostal.

A laparoscopia pode ser definida como a visualização telescópica da cavidade abdominopélvica (MACFADYEN \& PONSKY, 1992). Para GOMELLA\& STRUP (1994), a laparoscopia se caracteriza como o procedimento em que os conteúdos do peritônio são examinados com um endoscópio. FREEMAN (1998) acrescenta que a cirurgia endoscópica do abdome pode ser denominada igualmente laparoscopia, celioscopia ou pelvioscopia.

O caráter diagnóstico, que inicialmente impulsionou o acesso laparoscópico - sendo utilizado na identificação de doenças hepáticas, nas massas abdominais, nos diversos tipos de biópsias, na dor obscura e na isquemia mesentérica (SACKIER, 1992),

\footnotetext{
${ }^{1}$ Médico Veterinário, Doutor, Professor Assistente do Departamento de Medicina Animal da Faculdade de Veterinária, Universidade Federal do Rio Grande do Sul (UFRGS). Av. Bento Gonçalves, 9090, 91540-000, Porto Alegre, RS, Brasil. E-mail: afonso@orion.ufrgs.br

${ }^{2}$ Médico Veterinário, PhD, Professor Adjunto, Departamento de Clínica de Pequenos Animais, Universidade Federal de Santa Maria (UFSM).

${ }^{3}$ Médico Veterinário, Doutor, Professor do Curso de Medicina Veterinária, Universidade de Passo Fundo.

${ }^{4}$ Médico Veterinário, Mestre.

${ }^{5}$ Aluno do Curso de Mestrado em Medicina Veterinária, na área de Cirurgia Experimental, UFSM.

${ }^{6}$ Médico Veterinário, Doutor, Professor Adjunto, Departamento de Medicina Animal da Faculdade de Veterinária, UFRGS.

${ }^{7}$ Aluno do Curso de Medicina Veterinária, UFSM, Bolsista de Iniciação Científica.
} Recebido para publicação 05.06.03 Aprovado em 02.06.04 
ganha espaço a cada dia no tratamento de pacientes com dor abdominal crônica ou aguda, abdome agudo, peritonites e, mais recentemente, no trauma abdominal (BOYD \& NORD, 2000). Paralelamente, a partir da colicistectomia laparoscópica, o caráter terapêutico incorpora gradualmente novos procedimentos, após avaliações experimentais (STELLATO, 1992). Essas avaliações, muitas vezes, são utilizadas na comparação da técnica aberta convencional com a laparoscopia (GILL et al., 1998; SCHAUER et al., 1998) e, em outras ocasiões, na utilização de modelos experimentais em animais animais para testar o acesso (UNDERWOOD et al., 2000; GAMAL et al., 2001).

RICHTER (2001) relacionou, entre os órgãos avaliados com maior freqüência pela laparoscopia em cães e gatos, o fígado, o sistema biliar, o pâncreas, o rim, o baço, o intestino delgado, o intestino grosso e o trato genito-urinário. $\mathrm{O}$ autor destaca que, à semelhança do uso na medicina humana, o acesso laparoscópico na medicina veterinária pode ser empregado no diagnóstico do trauma abdominal para casos de lacerações hepáticas e esplênicas, rupturas da bexiga e do rim e em hérnias diafragmáticas.

Nos últimos anos, estudos utilizaram a laparoscopia na identificação de lesões diafragmáticas (MURRAY et al., 1998; MATZ et al., 2000; TORRESINI et al., 2000). MURRAY et al. (1998) realizaram laparoscopia em 110 pacientes com lesão penetrante do tórax esquerdo e hemodinamicamente estáveis. O estudo revelou que 26 pacientes possuíam lesões diafragmáticas e que $21 \%$ deles apresentaram exames clínicos e radiológicos sem anormalidades. Segundo MATZ et al. (2000), os pacientes com hérnias diafragmáticas são freqüentemente assintomáticos, podendo a hérnia permanecer clinicamente silenciosa por vários anos. Na opinião dos autores, a laparoscopia ou a toracoscopia pode identificar as lesões imediatamente após sua ocorrência.

Mais recentemente, a técnica passou a ser utilizada com finalidade terapêutica para correção de rupturas e de hérnias diafragmáticas (DOMENE et al., 1997; MEYER et al., 2000; KOZAR et al., 2001). DOMENE et al. (1997) relataram o êxito na correção de um caso de hérnia diafragmática, por meio do acesso laparoscópico, após três anos da lesão original. MEYER et al. (2000) descreveram a utilização da técnica laparoscópica em três casos de hérnias diafragmáticas - duas agudas e uma com mais de dez anos de ocorrência. A técnica mostrou-se viável nos três casos, com um tempo operatório médio de 93 minutos.

O presente estudo objetivou avaliar o acesso videolaparoscópico no diagnóstico e na terapêutica cirúrgica de hérnias diafragmáticas produzidas experimentalmente em cães.

\section{MATERIAL E MÉTODOS}

Foram utilizados oito cães adultos hígidos, machos, sem raça definida, oriundos do Biotério Central da UFSM, com pesos que variaram entre 12,5 e $21 \mathrm{~kg}$. Previamente à inclusão no experimento os animais foram avaliados clinicamente, everminados e alojados em canis coletivos contendo quatro cães, onde receberam, ração comercial e água ad libitum.

O experimento foi dividido em duas etapas. Na primeira etapa, os animais foram submetidos a um procedimento cirúrgico com o objetivo de induzir um modelo de hérnia diafragmática através do acesso laparoscópico. Em quatro cães, a indução da ruptura foi do lado direito e nos restantes do lado esquerdo. $\mathrm{O}$ protocolo anestésico constou de medicação préanestésica (MPA) com associação de cetamina (6,0mg $\left.\mathrm{kg}^{-1}\right)$ e midazolam $\left(0,3 \mathrm{mg} \mathrm{kg}^{-1}\right)$, via IM, na mesma seringa. Na indução anestésica, foi utilizada cetamina $\left(5,0 \mathrm{mg} \mathrm{kg}^{-1}\right)$, via IV, e, na manutenção, isofluorano vaporizado em oxigênio a $100 \%$ em circuito semifechado com respiração mecanicamente controlada.

A primeira etapa do experimento teve início com a punção realizada com a agulha de Veress, próxima à cicatriz umbilical e estabelecimento do pneumoperitônio utilizando uma pressão de $11 \mathrm{mmHg}$. Logo a seguir, foram realizadas as introduções dos três trocartes, respectivamente para a inserção do endoscópio e para os instrumentos de trabalho. Através de um redutor de diâmetro adaptado ao segundo trocarte, foi inserido um segmento de equipo com $6 \mathrm{~cm}$ de extensão, sendo este adaptado entre a porção esternal e costal do diafragma. Observando o sentido circunferencial foi realizada a demarcação do local da ruptura diafragmática. Com o objetivo de reduzir os riscos de um pneumotórax compressivo, o valor do pneumoperitônio foi diminuído de 11 para $5 \mathrm{mmHg}$, imediatamente antes de promover a ruptura do diafragma. Na seqüência, o defeito diafragmático previamente delimitado em $6 \mathrm{~cm}$, foi estabelecido com a utilização de uma tesoura de Metzenbaum. Ao final do procedimento, o instrumental cirúrgico e os trocartes foram removidos, os locais de punção foram obliterados e a pressão torácica negativa foi restabelecida por punção torácica com cateter venoso adaptado a uma válvula de três vias e seringa de $100 \mathrm{ml}$.

Após o período de uma semana, foi realizada a segunda etapa experimental, que constou inicialmente da identificação da ruptura diafragmática 
e/ou da presença de vísceras abdominais no tórax e, logo a seguir, a promoção da terapêutica cirúrgica de redução do conteúdo herniário e síntese do diafragma. $\mathrm{O}$ acesso utilizado foi igualmente o laparoscópico. $\mathrm{O}$ posicionamento da equipe cirúrgica, do animal, dos equipamentos e instrumental cirúrgico foi semelhante ao descrito na etapa de produção do defeito diafragmático (Figura 1A).

O pneumoperitônio foi promovido por meio do método aberto, com adaptação da cânula do $1^{\circ}$ trocarte através de pequena incisão de $2 \mathrm{~cm}$ de extensão, localizada sobre a linha média ventral, $1 \mathrm{~cm}$ cranial à cicatriz umbilical. A cânula foi fixada à parede abdominal por meio de uma sutura "em bolsa de fumo". A pressão previamente programada no insuflador automático de $\mathrm{CO}_{2}$ foi de $8 \mathrm{mmHg}$. Pelo fato de o procedimento visar a mesma estrutura anatômica, ou seja, o diafragma em sua porção previamente rompida, os locais de punção dos trocartes deveriam seguir os mesmos pontos utilizados na primeira etapa. Porém, como o intervalo entre as duas etapas foi de apenas uma semana e a cicatrização das incisões operatórias não se encontrava completa, optou-se por um deslocamento cranial de aproximadamente $3 \mathrm{~cm}$ em cada trocarte (Figura 1B).

A partir deste momento, teve início a exploração abdominal, denominada como procedimento diagnóstico. A localização da ruptura diafragmática e/ ou identificação de vísceras abdominais deslocadas para o tórax caracterizaram o procedimento como positivo. Além destes achados, foram registradas quaisquer alterações observadas no interior do abdome.

A laparoscopia teve seguimento com o início das manobras de tração e reposicionamento das vísceras abdominais. Nestas manobras, foram utilizadas pinça atraumática Babcock, pinça de Maryland, pinça de Kelly e afastador de vísceras articulado, através dos dois trocartes de trabalho. Após a redução visceral, iniciou-se a síntese da ruptura diafragmática. Um segmento de fio poliglactina 910 agulhado (no3-0), previamente medido em $20 \mathrm{~cm}$, foi introduzido no abdome por meio de um redutor de diâmetro, adaptado à cânula do $2^{\circ}$ trocarte. Com o uso de um porta-agulhas endoscópico e pinça de Kelly, foi realizada a síntese do diafragma, utilizando-se padrão de sutura contínua simples. Ao final, a cavidade foi inspecionada e as cânulas do $2^{\circ}$ e $3^{\circ}$ trocartes removidas. Os locais das duas punções foram obliterados por meio de sutura de Wolff com fio poliglactina-910 nํㅜ 3-0, para a musculatura abdominal e mononáilon 3-0 em padrão isolado simples para a pele. A entrada de $\mathrm{CO}_{2}$ foi suspensa, a cânula do $1^{\mathrm{o}}$ trocarte removida e o local da primeira punção foi suturado de maneira semelhante aos demais. O pneumotórax residual foi drenado utilizando a técnica descrita anteriormente.

\section{RESULTADOS}

O modelo de ruptura diafragmática mostrouse efetivo, permitindo o deslocamento visceral nos oito cães. O tempo cirúrgico total desta etapa variou

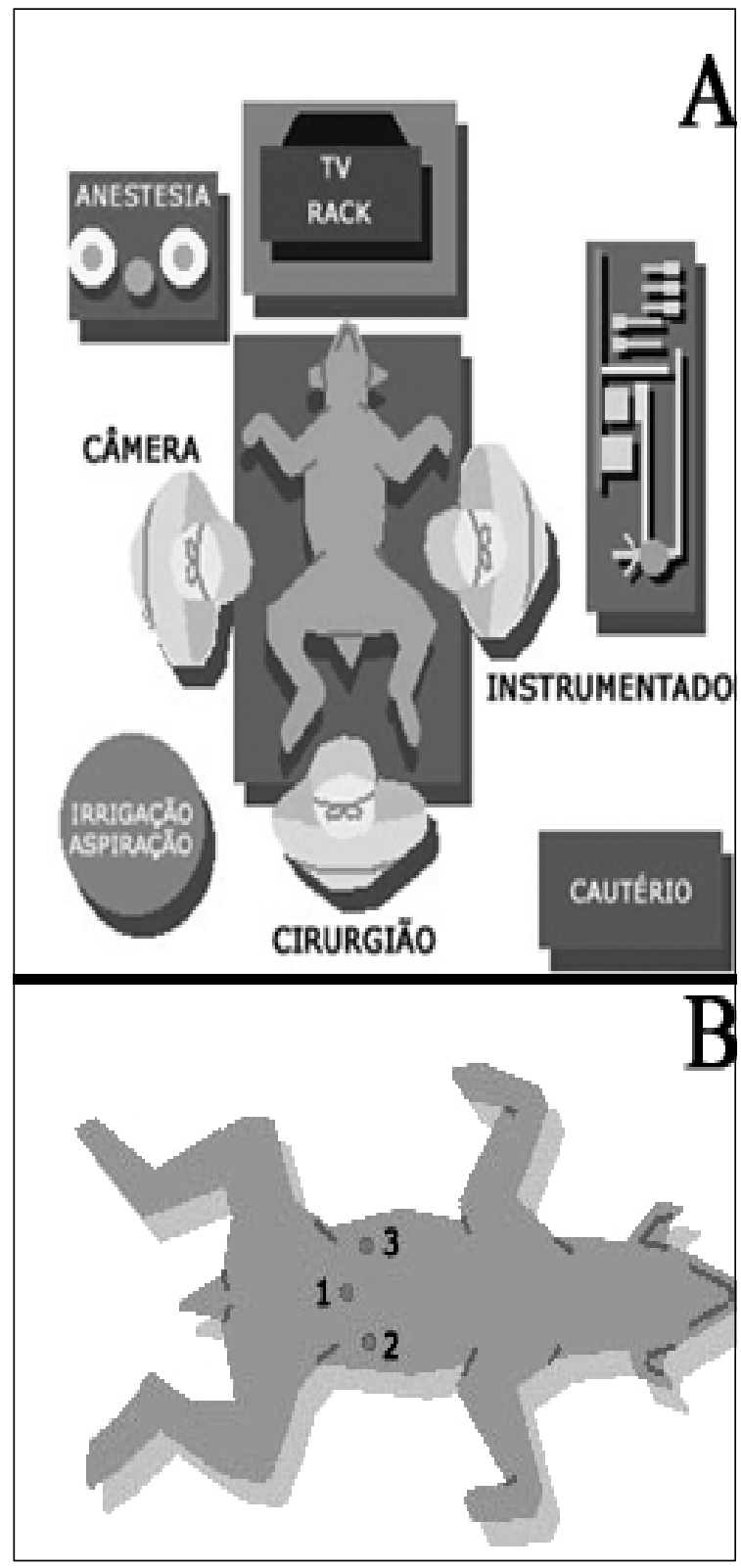

Figura 1 - Disposição da equipe cirúrgica e equipamentos durante os procedimentos laparoscópicos (A). Localização dos 3 trocartes em distribuição triangular durante as laparoscopias nos cães (B).

Ciência Rural, v.34, n.6, nov-dez, 2004. 
de 19 a 34 minutos, com tempo médio de 26 minutos. A abordagem possibilitou a visualização do diafragma, das estruturas e das vísceras abdominais. Não houve diferença entre os lados direito e esquerdo quanto à identificação do diafragma e à promoção de sua ruptura.

A introdução do segmento de equipo no interior do abdome mostrou-se fácil, utilizando-se um redutor de diâmetro no interior da cânula do segundo trocarte, para realizar tal tarefa. A redução da pressão de $\mathrm{CO}_{2}$ do pneumoperitônio, de $11 \mathrm{~mm}$ para $5 \mathrm{mmHg}$, antes da abertura diafragmática, evitou a ocorrência de pneumotórax compressivo durante as manobras cirúrgicas da $1^{\underline{a}}$ etapa. Durante o intervalo de uma semana entre a $1^{\mathrm{a}}$ e a $2^{\mathrm{a}}$ etapa, nenhum cão manifestou sinais de dispnéia, prostração ou inapetência.

As manobras relacionadas à $2^{\underline{a}}$ etapa do experimento resultaram em tempo cirúrgico total médio de 86,87 \pm 17,89 minutos. O acesso laparoscópico mostrou-se efetivo, permitindo a identificação da ruptura diafragmática, esquerda ou direita, nos oito cães. O posicionamento da equipe cirúrgica, bem como o da torre com os equipamentos vídeo-endoscópicos, o da mesa cirúrgica e o dos animais, mostrou-se adequado para a realização dos procedimentos cirúrgicos. A utilização do método aberto para introdução do primeiro trocarte foi satisfatória, sem a ocorrência de qualquer lesão iatrogênica durante a manobra. O pneumoperitônio, utilizando $8 \mathrm{mmHg}$ de $\mathrm{CO}_{2}$, foi eficaz, não ocorrendo alterações cardiorrespiratórias significantes sob o aspecto clínico, durante as oito intervenções.

A abordagem laparoscópica possibilitou uma adequada visualização de todo o diafragma, das estruturas e dos órgãos abdominais, além de permitir visibilização de boa parte do tórax, através da passagem do endoscópio pelo defeito diafragmático estabelecido. Não houve diferença relacionada ao acesso no que se refere aos lados direito e esquerdo da ruptura. $\mathrm{O}$ procedimento diagnóstico, realizado a partir da introdução do endoscópio, através da cânula do primeiro trocarte, foi considerado positivo para todos os cães. Entre as vísceras abdominais presentes no interior do tórax e de acordo com o número de animais, observou-se: o fígado (8), o omento (5), o intestino delgado (4), o pâncreas (1) e o ceco (1). Além do deslocamento das vísceras abdominais, foi possível identificar presença de efusão pleural (2), aderência do omento às bordas da lesão (1) e aderência de lobos hepáticos às bordas da lesão (5), presença de atelectasia pulmonar (2) (Figura 2A e 2B).

O fígado sofreu deslocamentos em todo os animais, variando apenas no número de lobos que ocuparam o tórax. Também quanto ao reposicionamento das vísceras deslocadas para o interior do abdome foi o fígado o órgão que apresentou maiores dificuldades.

A escolha dos três sítios de punção permitiu espaço adequado de manipulação do instrumental cirúrgico, com distância apropriada para a realização da reposição das vísceras abdominais e da síntese do diafragma em todos os oito cães. O procedimento terapêutico de reposicionamento das vísceras abdominais e síntese diafragmática foi viável nos oito cães deste grupo. Durante as manobras de redução visceral foi necessária a adoção do posicionamento de "Trendelenburg" reverso em quatro cães. Em dois desses animais, fez-se necessária a ampliação longitudinal da incisão prévia do diafragma em cerca de 1 a $2 \mathrm{~cm}$.

O fio poliglactina 910 no 3-0 agulhado, previamente medido em $20 \mathrm{~cm}$, mostrou-se adequado tanto pela facilidade de manipulação no interior do abdome quanto pela resistência na realização da síntese da ruptura do diafragma. O padrão de sutura contínua simples revelou-se igualmente satisfatório, permitindo boa aposição das bordas da ferida diafragmática, além de assegurar a resistência desejada (Figura 2C).

\section{DISCUSSÃO}

A prévia organização e planejamento no posicionamento da torre com os equipamentos endoscópicos, do equipamento anestesiológico e do anestesista, da equipe cirúrgica, da mesa de instrumental e do animal, proporcionou espaço de trabalho adequado, boa visibilidade do monitor e fácil fluxo de pessoal no interior da sala operatória durante os procedimentos cirúrgicos do experimento. Este planejamento inicial é destacado por FREEMAN (1998) como fator importante para o êxito cirúrgico nos procedimentos videoendoscópicos.

O modelo de hérnia diafragmática proposto no atual trabalho mostrou-se eficiente, além de demonstrar fácil e rápida execução, promovendo mínimo trauma cirúrgico. Verificou-se o deslocamento de vísceras e/ou estruturas abdominais nos oito cães do experimento, sem a ocorrência de nenhum óbito no trans e no pós-operatório. Na revisão de literatura, foram encontrados dois trabalhos que utilizaram a cirurgia videoendoscópica para a produção de defeito diafragmático em animais. No primeiro, foram avaliadas as alterações decorrentes do pneumotórax durante intervenções laparoscópicas após a promoção de um pequeno orifício no diafragma esquerdo de suínos (MARCUS et al., 1996). No outro estudo, os autores utilizaram o acesso toracoscópico para estabelecer uma 

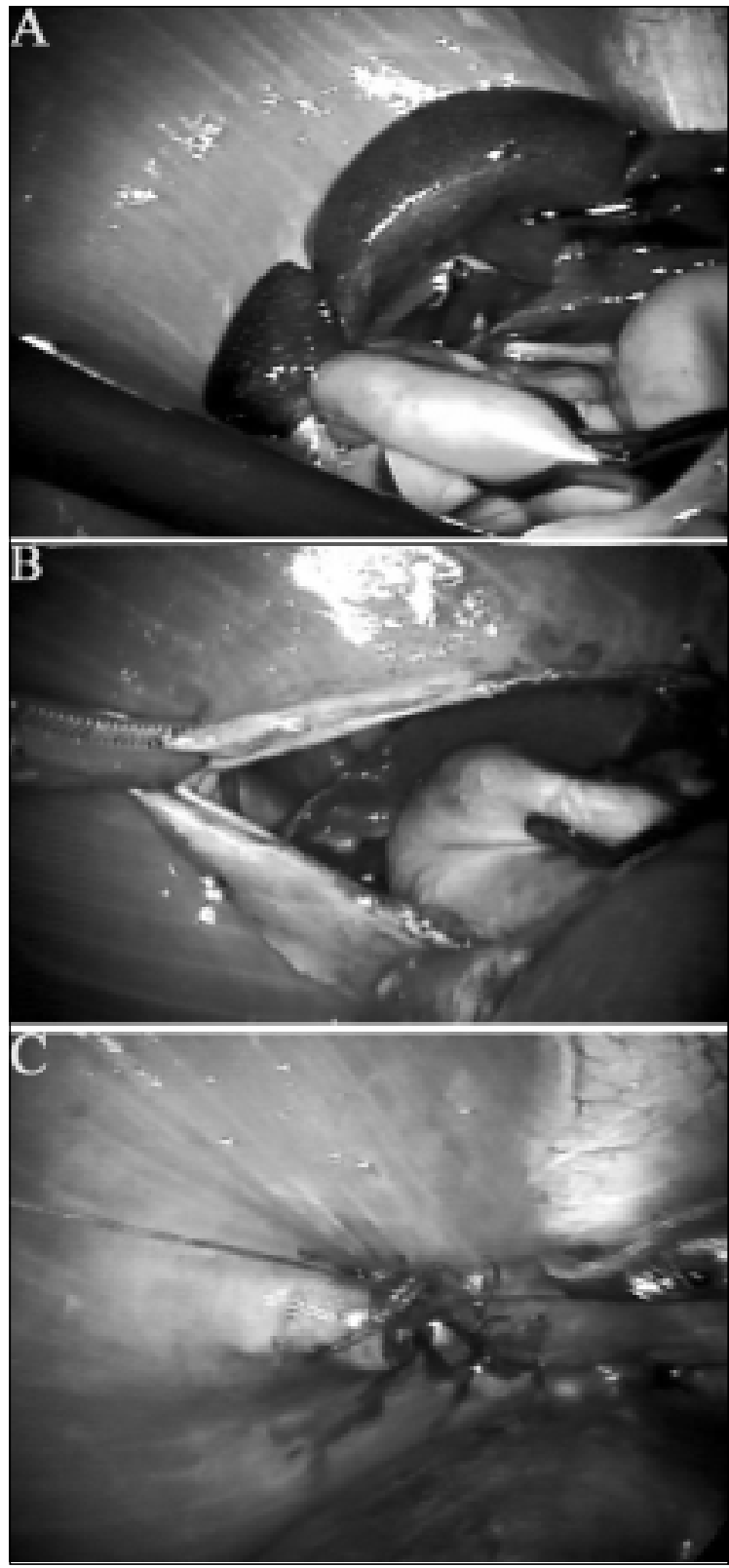

Figura 2 - Imagem laparoscópica revelando: deslocamento de vísceras abdominais para o interior do tórax as hérnias diafragmáticas $(\mathrm{A})$; tração de lobo hepático deslocado (B); drenagem de efusão pleural; sutura em padrão contínuo simples do diafragma (C).

ruptura diafragmática em suínos com o objetivo de avaliar e comparar os exames de ultra-sonografia e de radiografia como métodos de diagnóstico (ZIEROLD et al., 2001).

A redução da pressão do peumoperitônio de 11 para $5 \mathrm{mmHg}$, antes de proceder a abertura do diafragma, na etapa de preparo do modelo de hérnia diafragmática, teve como objetivo reduzir as chances de ocorrência de pneumotórax compressivo. Tal aspecto é destacado por MARCUS et al. (1996), ao afirmarem que as alterações provocadas por um pneumotórax produzido durante uma cirurgia videoendoscópica podem ser graves.

No presente estudo, todos os cães submetidos à segunda etapa operatória apresentavam estabilidade hemodinâmica, sendo este um aspecto considerado fundamental em estudos que utilizaram a cirurgia videoendoscópica na terapêutica de hérnias diafragmáticas em seres humanos (LOWDERMILK \& NAUNHEIM, 2000; MARTINEZ et al., 2001).

Os resultados encontrados na literatura são controversos no que se refere aos achados diagnósticos da laparoscopia no trauma abdominal e torácico em humanos. BERCI et al. (1991), após a realização de 150 minilaparoscopias em pacientes com trauma abdominal contuso, obtiveram índices de diagnóstico com eficácia próxima a 100\%. ORTEGA et al. (1996) consideram que o acesso laparoscópico é positivo na violação peritoneal, nas lesões diafragmáticas e nas lesões a órgãos sólidos, mas não apresenta bons resultados nas lesões a vísceras ocas. No atual estudo, a utilização diagnóstica do acesso laparoscópico durante a segunda etapa do experimento permitiu a identificação da ruptura diafragmática e do deslocamento das vísceras e estruturas abdominais em todos os cães, sem diferença quanto ao lado do defeito.

A laparoscopia permitiu a observação de todo o diafragma através de um único acesso. $\mathrm{Na}$ exploração diagnóstica de casos clínicos, em animais com suspeita de ruptura diafragmática, este aspecto pode ser considerado importante na escolha da técnica endoscópica a ser utilizada, já que a ruptura afeta igualmente ambos os lados do diafragma dos cães (WILSON et al.,1971) e nem sempre o local da ruptura pode ser identificado radiograficamente (HAGE \& IWASAKI, 2001).

A possibilidade demonstrada pela laparoscopia, permitindo a exploração de ambas as cavidades, através do defeito diafragmático, indica uma vantagem importante deste acesso sobre a laparotomia mediana. Os estudos encontrados na literatura consideram a laparotomia mediana como a abordagem mais indicada para os casos de hérnias diafragmáticas em cães e gatos (WILSON et al., 1971; LEVINE, 1987). Destaca-se, todavia, que esta abordagem apresenta como desvantagens, o fato de não permitir a visualização da cavidade torácica e a lise segura das aderências entre as vísceras abdominais e as estruturas torácicas. Por isso, em alguns casos, torna-se necessária a extensão da laparotomia até uma esternotomia mediana 
(JOHNSON, 1993). Tal procedimento ocasiona não apenas acréscimo nas dificuldades relacionadas ao ato cirúrgico, mas maior morbidade e desconforto pósoperatório aos pacientes. SACKIER (1992) sublinha a importância dos procedimentos minimamente invasivos nesses casos, uma vez que descartam a prática de cirurgias amplas, com complicações potenciais e, pior, realizadas muitas vezes sem necessidade. $\mathrm{O}$ presente estudo abre espaço para a utilização diagnóstica do acesso videolaparoscópico nos casos de trauma toracoabdominal em cães.

A viabilidade da redução do conteúdo herniado nos oito cães foi certamente facilitada pelo intervalo de uma semana observado entre a primeira e a segunda etapas cirúrgicas do experimento. $\mathrm{O}$ intervalo proposto limita a formação de aderências maduras com maiores níveis de tecido fibrovascular organizado, que, segundo JOHNSON (1993), inicia-se a partir da segunda semana após o trauma.

A utilização do posicionamento em "Trendelenburg reverso" em quatro cães, facilitou o reposicionamento das vísceras abdominais deslocadas. SULLIVAN \& REID (1990) indicaram este posicionamento em laparotomias realizadas em cães e gatos acometidos por hérnias diafragmáticas. Outra manobra cirúrgica recomendada para estes casos é a ampliação da ruptura diafragmática (KAGAN, 1980; SULLIVAN \& REID, 1990; JOHNSON, 1993). No atual estudo, tal manobra foi realizada em dois cães durante os procedimentos da segunda etapa cirúrgica, facilitando a redução visceral.

\section{CONCLUSÕES}

O êxito na utilização da videolaparoscopia, para o diagnóstico e terapêutica cirúrgica de hérnias diafragmáticas produzidas experimentalmente em cães, sugere seu uso e avaliação em casos clínicos de pacientes estáveis e com tempo de ruptura diafragmática de até uma semana.

\section{REFERÊNCIAS BIBLIOGRÁFICAS}

BERCI, G. et al. Emergency laparoscopy. Am J Surg, New York, v.161, p.332-335, 1991.

BOUDRIEAU, R.J.; MUIR, W.W. Pathophysiology of traumatic diaphragmatic hérnia in dogs. Comp Cont Educ Pract Vet, Toronto, v.9, n.4, p.379-385, 1987.

BOYD Jr, W.P.; NORD, H.J. State-of-art review - Diagnostic laparoscopy. Endoscopy, New York, v.32, n.2, p.153-158, 2000.

DOMENE, C.E. et al. Correção laparoscópica de hérnia diafragmática traumática crônica: relato de caso e revisão de literatura. Rev Hosp Clin Fac Med, São Paulo, v.52, n.5, p.271-275, 1997.
FREEMAN, L.J. Introduction. In: FREEMAN, L.J. Veterinary endosurgery. St Louis : Mosby, 1998. p.xxxvii.

GAMAL, E.M. et al. The influence of intraoperative complications on adhesion formation during laparoscopic and conventional cholecystectomy in an animal model. Surg Endosc, New York, v.15, n.8, p.873-877, 2001.

GILL, I.S. et al. Needlescopiccadrenalectomy - the initial series: comparison with conventional laparoscopic adrenalectomy. Urology, New York, v.52, n.2, p.180-186, 1998

GOMELLA, L.G.; STRUP, S.E. The history of urologic laparoscopy: from cystoscope to laparoscope. In: GOMELLA, L.G. et al. Laparoscopic urologic surgery. New York : Raven, 1994. 286p. p.9-14.

HAGE, M.C.F.N.S.; IWASAKI, M. Contribuição ao estudo radiográfico das rupturas diafragmáticas em cães e gatos. Clínica Veterinária, São Paulo, n.35, p.36-50, 2001.

JOHNSON, K.A. Diaphragmatic, pericardial, and hiatal hernia. In SLATTER, D. Textbook of small animal surgery. 2.ed. Philadelphia : Saunders, 1993. V.1. p.455-470.

KAGAN, K.G. Thoracic trauma. Vet Clin North Am: Small Anim Pract, Philadelphia, v.10, n.3, p.641-653, 1980.

KOZAR, R.A. et al. Laparoscopic repair of traumatic diaphragmatic injuries. J Surg Research, New York, v.97, n.2, p.164-171, 2001.

LEVINE, S.H. Diaphragmatic hernia. Vet Clin North Amer: Small Anim Pract, Philadelphia, v.17, n.2, p.411-430, 1987.

LOWDERMILK, G.A.; NAUNHEIM, K.S. Thoracoscopic evaluation and treatment of thoracic trauma. Surg Clin North Am, Philadelphia, v.80, n.5, p.1535-1542, 2000.

MACFADYen, B.V.; PONSKY, J.L. Prefácio. Clin Cir Am Norte, v.5, p.xiii, 1992

MARCUS, D.R.; LAU,W.M.; SWANSTROM, L.L. Carbon dioxide pneumothorax in laparoscopic surgery. Am J Surg, New York. v.171, n.5, p.464-466, 1996.

MARTINEZ, M. et al. Surg Endosc, New York, v.15, p.28$32,2001$.

MATZ, A. et al. The role of laparoscopy in the diagnosis and treatment of missed diaphragmatic ruptures. Surg Endosc, New York, v.14, n.6, p.537-539, 2000.

MEYER, G. et al. Laparoscopic repair of traumatic diaphragmatic hernias. Surg Endosc, New York, v.14, n.11, p.1010-1014, 2000.

MURRAY, J.A. et al. Occult injuries to the diaphragm: prospective evaluation of laparoscopy in penetrating injuries to the left lower chest. J Am Coll Surg, New York, v.187, n.6, p.626-630, 1998.

ORTEGA, A.E. et al. Laparoscopic evaluation of penetrating thoracoabdominal traumatic injuries. Surg Endosc, New York, v.10, n.1, p.19-22, 1996. 
RAISER, A.G. Hérnias em cães e gatos - análise de 188 casos e proposição de técnica de herniorrafia para ruptura costal do diafragma. 1993. 108f. Tese (Concurso para Professor Titular) - Universidade Federal de Santa Maria.

RICHTER, K.P. Laparoscopy in dogs and cats. Vet Clin North Am: Small Anim Pract, Philadelphia, v.31, n.4, p.707-727, 2001.

SACKIER, J. Laparoscopia diagnóstica na doença não-maligna. Clin Cir Am Norte, Rio de Janeiro, v.5, p.1051-1060, 1992.

SCHAUER, P.R. et al. Comparison of laparoscopic versus open repair of peraesophageal hernia. Am J Surg, New York, v.176, p.659-665, 1998.

STEllato, T.A. História da cirurgia laparoscópica. Clin Cir Am Norte, Rio de Janeiro, v.5, p.1015-1020, 1992

SULLIVAN, M.; REID, J. Management of 60 cases of diaphragmatic rupture. J Small Anim Pract, Philadelphia, v.31, n. 9, p.425-430, 1990 .

TORRESINI, G. et al. Laparoscopic repair of diaphragmatic iatrogenic hernia. Endoscopy, New York, v.32, p.S2, 2000 .

UNDERWOOD, R.A. et al. Development of laparoscopic approach to neurolytic celiac plexus block in a porcine model. Surg Endosc, New York, v.14, n.9, p.839-843, 2000.

WILSON, G. P. et al. A review of 116 diaphragmatic hérnias in dogs and cats. J Am Vet Med Assoc, Chicago, v.159, n.9, p.1142-1145, 1971

ZIEROLD, D. et al. Penetrating trauma to the diaphragm. Natural history and ultrasonographic characteristics of untreated injury in a pig model. Arch Surg, New York, v.136, p.32-37, 2001. 